\title{
Vaccination strategies and backward bifurcation in an age-since-infection structured model
}

\author{
Christopher M. Kribs-Zaleta* Maia Martcheva ${ }^{\dagger}$
}

\begin{abstract}
We consider models for a disease with acute and chronic infective stages, and variable infectivity and recovery rates, within the context of a vaccination campaign. Models for SIRS and SIS disease cycles exhibit backward bifurcations under certain conditions, which complicate the criteria for success of the vaccination campaign by making it possible to have stable endemic states when $\mathcal{R}_{0}<1$. We also show the extent to which the forms of the infectivity and recovery functions affect the possibility of backward bifurcations. SIR and SI models examined do not exhibit this behavior.
\end{abstract}

Key words: backward bifurcation, variable infectivity, vaccination, SIRS model with chronic stage, age-since-infection structure.

\section{Introduction}

This paper studies the effects of a vaccination campaign upon the spread of a nonfatal disease which features both acute and chronic infective stages, as well as variable infectivity and recovery rates in the chronic stage. Diseases which are compatible with this description include hepatitis A and $\mathrm{B}$ as well as feline calicivirus (FCV) [19], which causes upper respiratory tract infections in cats and features an asymptomatic chronic stage in which pathogen shedding continues. Vaccination campaigns have been critical in attacking the spread of several diseases, most notably smallpox. An efficient vaccination campaign acts to reduce the disease's basic reproductive number $\mathcal{R}_{0}$ below the critical level of 1 , where, in most simple models, only the disease-free equilibrium exists. Recent models on the transmission dynamics of diseases such as HIV and tuberculosis have shown, however, that the $\mathcal{R}_{0}<1$ criterion is not always sufficient to control the spread of a disease when the at-risk population is divided into classes with different susceptibilities to the disease, e.g., [2, 3, 4, 7, 8, 9, $10,12,17$. Under certain conditions, a stable endemic state may exist even when $\mathcal{R}_{0}<1$, usually due to a phenomenon known as a "backward" bifurcation; in such cases, $\mathcal{R}_{0}$ must be reduced well below 1 to ensure eradication of the disease. Variation in infectivity is also important to model when the disease's timescale is on the same order as the demographic timescale [14], as evidenced by the worldwide AIDS epidemic. Some diseases feature an initial infective period when symptoms and infectivity are high, followed by a low-level or chronic stage during which the body has already begun to mount an immune response to the infection, and symptoms and infectivity are sharply reduced. Infectivity and recovery rates may continue to vary during this latter stage.

\footnotetext{
*Department of Mathematics, University of Texas at Arlington, Arlington, TX, 76019, kribs@uta.edu

${ }^{\dagger}$ Department of Mathematics, Polytechnic University, Six MetroTech Center, Brooklyn, NY 11201, mayam@duke.poly.edu, (corresponding author).
} 
The stable endemic state introduced for $\mathcal{R}_{0}<1$ arises in dynamical systems theory when the transcritical bifurcation (exchange of stability) that occurs at $\mathcal{R}_{0}=1$ changes directions, and the endemic equilibrium arises from the disease-free equilibrium for $\mathcal{R}_{0}<1$ rather than $\mathcal{R}_{0}>1$. In this case, it is possible for the disease to establish itself in a population, given a sufficiently large initial outbreak, in conditions which would not normally permit it. Alternatively, once a disease has become endemic in a population, it is much more difficult to eradicate if the bifurcation at $\mathcal{R}_{0}=1$ is backward: $\mathcal{R}_{0}$ must be reduced below the leftmost point on the bifurcation curve for which an endemic equilibrium exists, $\mathcal{R}_{0}^{c}$, sometimes called the minimum transition value [20]. Mathematically speaking, when a backward bifurcation occurs and $\mathcal{R}_{0}^{c}<\mathcal{R}_{0}<1$, there are at least three equilibria: the stable disease-free equilibrium, a large stable endemic equilibrium, and a small unstable endemic equilibrium which acts as a boundary between the basins of attraction for the two stable equilibria. Epidemiologically speaking, it has been observed (e.g., [10, 17]) that backward bifurcations tend to arise when the population at risk is divided into groups with different susceptibilities to the disease - for instance, different contact rates [2, 12] (including by age), having been previously infected [4], or vaccinated and unvaccinated classes [7, 17], or superinfection of already infected individuals [3].

Although many disease models assume constant per capita rates for all transitions, the forms of the functions describing passage from one stage of a disease to another have been seen to have important effects on long-term behavior of the models in some cases. Among others, Brauer [1], Kim \& Milner [14] and Thieme \& Castillo-Chavez [18] have studied the effects of variable infectivity on the spread of infectious diseases. The variable infectivity of HIV/AIDS in particular has had a tremendous impact on the shape of the AIDS epidemic which continues to skyrocket in many countries worldwide, and has rebounded following earlier attempts to contain it. The studies cited above found that variable infectivity in some (mostly SIR-type) models could destabilize the endemic equilibrium which exists for $\mathcal{R}_{0}>1$, leading to sustained oscillations, rather than a stable endemic level, under certain conditions. In this study we are interested in the possible effects of variable infectivity on the long-term behavior of a disease when $\mathcal{R}_{0}<1$ due to a vaccination campaign.

In the models developed below, therefore, we will suppose that the vaccine being administered is effectively permanent and provides complete protection against low-level infection from chronicstage individuals, but only partial protection against acutely infective contacts. We begin our investigation in Section 2 with a model for an SIRS disease cycle, whose analysis will serve as a basis for a number of other models in Section 3 which are special cases of it. In Section 4 we examine the effects of variable infectivity and recovery rates. We conclude with a discussion of the results.

\section{An SIRS model}

We shall now consider an SIRS model with both acute and chronic infective stages, as well as a vaccination program. We will suppose the vaccine's effects to be permanent (it does not wear off) but only proportionally effective (it only "takes" for some of the population, and so reduces the overall rate of infection by a given factor). In keeping with infections such as hepatitis A and B, and some fungal infections, we will also suppose that the acute stage of infection is considerably more infectious than the chronic stage. In fact, we will assume that the vaccine not only reduces the overall likelihood of infection by an acute infective but eliminates the possibility of infection by chronic-stage infectives. Infected individuals always pass through both stages before recovery - that is, treatment can only be administered effectively in the chronic stage — but chronic-stage 
infectives have an infectivity that varies with time spent in that stage, as does their recovery rate. All other rates are assumed constant, except for the infection terms, which follow the customary mass action law. We incorporate demographics external to the disease via constant per capita birth and death rates, and assume that all newborns are susceptible.

We therefore structure the population into five compartments: susceptibles $S$, vaccinated $V$, acute infectives $I$, chronic infectives $C$, and recovered (temporarily immune) individuals $R$. We further classify chronic infectives by age of [chronic] infection $\theta$ to obtain $\chi(\theta, t)$, with $C(t)=$ $\int_{0}^{\infty} \chi(\theta, t) d \theta$, and a total population $N$, with $S+V+I+C+R=N$.

We can now write the model

$$
\begin{aligned}
S^{\prime} & =\quad b N-\mu S-\gamma \frac{S}{N} I-\frac{S}{N} \int_{0}^{\infty} \delta(\theta) \chi(\theta, t) d \theta-\psi S+c R \\
V^{\prime} & =\psi S-\sigma \gamma \frac{V}{N} I-\mu V \\
I^{\prime} & =\gamma\left(\frac{S}{N}+\sigma \frac{V}{N}\right) I+\frac{S}{N} \int_{0}^{\infty} \delta(\theta) \chi(\theta, t) d \theta-(\mu+k) I \\
\chi_{\theta}+\chi_{t} & =-(\mu+\alpha(\theta)) \chi(\theta, t) ; \chi(0, t)=k I \\
R^{\prime} & =\int_{0}^{\infty} \alpha(\theta) \chi(\theta, t) d \theta-(\mu+c) R
\end{aligned}
$$

where $b$ is the per capita birth rate,

$\mu$ is the per capita death rate (unrelated to disease),

$\psi$ is the per capita vaccination rate,

$\sigma$ is the factor by which the vaccine reduces the likelihood of infection by acute infectives,

$\gamma$ is the effective per capita infection rate for acute infectives,

$\delta(\theta)$ is the per capita infection rate for chronic infectives with infection age $\theta$,

$k$ is the per capita rate at which acute infectives progress into the chronic stage,

$\alpha(\theta)$ is the per capita treatment/recovery rate for chronic infectives with infection age $\theta$, and $c$ is the per capita rate for loss of immunity (return to susceptibility).

Here $\sigma=0$ for a perfect vaccine and 1 for a useless one; in general $0 \leq \sigma \leq 1$. We assume that all parameters are nonnegative, with $b>0, \mu>0, \gamma>0$, and that $\alpha(\theta), \delta(\theta)$ are bounded functions of $\theta$. We will also assume that $\int_{0}^{\infty} \alpha(\theta) d \theta=\infty$, in order to guarantee that for all $t$

$$
\lim _{\theta \rightarrow \infty} \chi(\theta, t)=0 .
$$

In addition, we can specify initial conditions $S(0)=S_{0}$, etc.

The model is homogeneous (of degree one). We obtain the equation for the total population size by summing the equations for the population subclasses:

$$
N^{\prime}=b N-\mu N .
$$

This equation has the solution $N(t)=N_{0} e^{(b-\mu) t}$. Thus $(b-\mu)$ gives the growth rate of the population; if $b>\mu$, the population is growing exponentially, and if $b<\mu$, the population is decreasing exponentially. The population is stationary if $b=\mu$.

We also note that we can write an equation for the chronic class:

$$
C^{\prime}=k I-\mu C-\int_{0}^{\infty} \alpha(\theta) \chi(\theta, t) d \theta .
$$

We will now normalize the model to consider the proportional dynamics of the system. We define $s=S / N, v=V / N, i=I / N, u=\chi / N, r=R / N$, so that 


$$
s+v+i+\int_{0}^{\infty} u(\theta, t) d \theta+r=1
$$

and rewrite model $(1)$, substituting $N^{\prime}=(b-\mu) N$ :

$$
\begin{aligned}
s^{\prime} & =b(1-s)-\gamma s i-s \int_{0}^{\infty} \delta(\theta) u(\theta, t) d \theta-\psi s+c r \\
v^{\prime} & =\psi s-\sigma \gamma v i-b v \\
i^{\prime} & =\gamma i(s+\sigma v)+s \int_{0}^{\infty} \delta(\theta) u(\theta, t) d \theta-(b+k) i \\
u_{\theta}+u_{t} & =-(b+\alpha(\theta)) u(\theta, t) ; \quad u(0, t)=k i \\
r^{\prime} & =\int_{0}^{\infty} \alpha(\theta) u(\theta, t) d \theta-(b+c) r
\end{aligned}
$$

This model is not homogeneous, but it has steady states in the classical sense. If $\left(s^{*}, v^{*}, i^{*}, u^{*}(\theta), r^{*}\right)$ is a steady state of model $(3)$, then $\left(s^{*}, v^{*}, i^{*}, u^{*}(\theta), r^{*}\right) e^{(b-\mu) t}$ is a "steady state" of model (1) in the sense that all corresponding solutions are expected to approach it in time (cf. $[5,6,11])$. The disease-free equilibrium (henceforth DFE) of (3) is

$$
s^{*}=\frac{b}{b+\psi}, \quad v^{*}=\frac{\psi}{b+\psi}, \quad i^{*}=0, \quad u^{*}(\theta)=0, \quad r^{*}=0 .
$$

There are several ways to determine the infection's reproductive number. We shall first compute it as a threshold parameter which determines the local stability of the DFE, and then interpret it epidemiologically. In particular, we now linearize system (3) about the DFE and look for eigenvalues of the linear operator - that is, we look for solutions where each state variable has the form $K_{j} e^{\lambda t}$, where the $K_{j}$ are distinct constants (a function of $\theta$ in the case of $u$ ) but the eigenvalue $\lambda$ is common. This process results in the following characteristic equation:

$$
\lambda+b+k=\gamma \frac{b+\sigma \psi}{b+\psi}+\frac{b}{b+\psi} k \int_{0}^{\infty} \delta(\theta) e^{-(\lambda+b) \theta} \pi(\theta) d \theta
$$

where $\pi(\theta) \equiv e^{-\int_{0}^{\theta} \alpha(\eta) d \eta}$ is the probability of not having recovered $\theta$ units of time after entering the chronic class.

If we consider $\lambda$ real, the expression on the left hand side of (5) increases linearly in $\lambda$ and is negative for $\lambda<-(b+k)$. The expression on the right hand side is a monotone decreasing function of $\lambda$ which approaches $\infty$ as $\lambda \rightarrow-\infty$ and zero as $\lambda \rightarrow \infty$. This implies that equation (5) has a unique real solution $\lambda>-(b+k)$. We can also verify that the real part of any complex solution of (5) does not exceed the unique real solution. Indeed, if $\lambda$ is a complex solution of (5) then its real part, $\Re \lambda$, satisfies the inequality

$$
\Re \lambda+b+k \leq \gamma \frac{b+\sigma \psi}{b+\psi}+\frac{b}{b+\psi} k \int_{0}^{\infty} \delta(\theta) e^{-(\Re \lambda+b) \theta} \pi(\theta) d \theta .
$$

We introduce the notation

$$
\mathcal{G}(\lambda)=\frac{\gamma}{\lambda+b+k} \frac{b+\sigma \psi}{b+\psi}+\frac{b}{b+\psi} \frac{k}{\lambda+b+k} \int_{0}^{\infty} \delta(\theta) e^{-(\lambda+b) \theta} \pi(\theta) d \theta .
$$

Clearly, $\mathcal{G}(\lambda)=1$ for every solution $\lambda$ of (5). Let $\lambda^{*}$ be the unique real solution. Then by (6), $\mathcal{G}\left(\lambda^{*}\right)=1 \leq \mathcal{G}(\Re \lambda)$. Consequently, since $\mathcal{G}$ is decreasing for $\lambda>-(b+k), \Re \lambda \leq \lambda^{*}$. 
To simplify notation, we now define

$$
d \equiv \int_{0}^{\infty} \delta(\theta) e^{-b \theta} \pi(\theta) d \theta
$$

the average number of infectious contacts made by an individual while in the chronic stage. Here $e^{-b \theta}$ is the probability of surviving to infection age $\theta$, and $\pi(\theta)$ is, as stated above, the probability of not having recovered by infection age $\theta$ [13]. Now we can introduce the infection's reproductive number, and denote it by $\mathcal{R}(\psi)$ to show that we consider it to depend on the vaccination policy $\psi$ :

$$
\mathcal{R}(\psi)=\frac{b}{b+\psi} \frac{\gamma+k d}{b+k}+\frac{\psi}{b+\psi} \frac{\sigma \gamma}{b+k} .
$$

If $\mathcal{R}(\psi)<1$, then for $\lambda=0$ the right hand side of (5) is smaller than the left hand side, so that the real solution must satisfy $\lambda<0$. In this case the DFE is locally asymptotically stable. However, for $\mathcal{R}(\psi)>1$, the right hand side is larger, and the real solution satisfies $\lambda>0$, making the DFE locally unstable.

To construct $\mathcal{R}(\psi)$ from epidemiological principles, recall that the basic reproductive number represents the average number of secondary infections caused by a single infective in a population of susceptibles (and vaccinated). In this case, a proportion $\frac{b}{b+\psi}$ of the contacts made would be with susceptibles, and $\frac{\psi}{b+\psi}$ of the contacts made would be with vaccinated individuals. An average infective will make $\frac{\gamma}{b+k}$ infectious contacts while in the acute stage; this number is reduced by a factor of $\sigma$ with regard to infecting vaccinated individuals. In addition, a proportion $\frac{k}{b+k}$ of infectives progress to the chronic stage before dying, and those infectives then make an average of $d$ infectious contacts while in the chronic stage. We can therefore interpret equation (8) as the sum of the proportions of infections made in the ways described above.

We observe that $\mathcal{R}(\psi)$ is a decreasing function of $\psi$, which reflects the expectation that the reproductive number of the disease becomes smaller under the application of a vaccination campaign. We note that $\mathcal{R}(0)$ is the [basic] reproductive number in the absence of vaccination; we will denote $\mathcal{R}(0)$ by $\mathcal{R}_{0}$. Note that the rate $c$ at which immunity is lost does not affect $\mathcal{R}(\psi)$.

One can write $\mathcal{R}(0)$ as a sum of two reproductive numbers: $\mathcal{R}(0)=\mathcal{R}_{I}(0)+\mathcal{R}_{C}(0)$ where $\mathcal{R}_{I}(0)=\frac{\gamma}{b+k}$ is the reproductive number corresponding to infectious individuals, while $\mathcal{R}_{C}(0)=\frac{k d}{b+k}$ is the reproductive number corresponding to chronic individuals. In addition we mention that $\mathcal{R}(\psi)$ and $\mathcal{R}_{0}$ are the reproductive numbers for proportions rather than number of individuals.

We now turn our attention to the endemic equilibria of (3). If we write the equilibrium conditions, by setting the time derivatives to zero, we can express $s^{*}, v^{*}, u^{*}(\theta)$ and $r^{*}$ in terms of $i^{*}$ recursively from the following expressions:

$$
\begin{aligned}
& u^{*}(\theta)=k i^{*} e^{-b \theta} \pi(\theta) \\
& r^{*}=\frac{k i^{*}}{b+c} \int_{0}^{\infty} \alpha(\theta) e^{-b \theta} \pi(\theta) d \theta \\
& s^{*}=\frac{b+c r^{*}}{b+\psi+(\gamma+k d) i^{*}} \\
& v^{*}=\frac{\psi s^{*}}{\sigma \gamma i^{*}+b}
\end{aligned}
$$

We can factor out the DFE and eliminate $s^{*}, v^{*}, u^{*}(\theta)$ and $r^{*}$, to obtain a single quadratic equation for $i^{*}$, 


$$
A i^{2}+B i+C=0
$$

where $^{1}$

$$
\begin{aligned}
& A=\sigma \gamma(\gamma+d k)\left(1+\frac{k}{b+c}(1+c \tau)\right) \geq 0, \\
& B=(b(\gamma+d k)+\sigma \gamma \psi)\left(1+\frac{k}{b+c}(1+c \tau)\right)+\sigma \gamma(b+k-(\gamma+d k)), \\
& C=(b+k)(b+\psi)-(b(\gamma+d k)+\sigma \gamma \psi)=(b+k)(b+\psi)[1-\mathcal{R}(\psi)],
\end{aligned}
$$

and the average duration spent in the chronic stage (given entry) is given by

$$
\tau \equiv \int_{0}^{\infty} e^{-b \theta} \pi(\theta) d \theta
$$

We note that $A=0$ if and only if $\sigma=0$. If $A=0$ then the endemic equilibrium is unique and exists only for $\mathcal{R}(\psi)>1$. In what follows we assume $\sigma>0$.

In the case that $\mathcal{R}(\psi)>1$, we can see that $C<0$, so that the discriminant $B^{2}-4 A C$ is positive and exceeds $B^{2}$. There is therefore exactly one positive solution. If, however, $\mathcal{R}(\psi)<1$, then $C>0$, and (10) has either zero or two positive solutions. In order to have two positive solutions we need $B^{2}-4 A C>0$ and $B<0$ (so that $-\frac{B}{2 A}>0$ ). [Note that we can write all three conditions as $B+2 \sqrt{A C}<0$.]

Proposition 1. Assume $\sigma>0$. If $\mathcal{R}(\psi)<1, B<0$, and $B^{2}-4 A C>0$, then system (3) has multiple endemic equilibria.

Proof. Since $B^{2}-4 A C>0, A C>0$ and $B<0$, the quadratic equation (10) has two distinct real positive solutions. It remains to show that if (10) has two positive solutions, they both automatically satisfy $i<1$. We now observe that $-B<\sigma \gamma(\gamma+d k)$, so that

$$
-\frac{B}{2 A}<\frac{\sigma \gamma(\gamma+d k)}{2 \sigma \gamma(\gamma+d k)\left(1+\frac{k}{b+c}(1+c \tau)\right)} \leq \frac{1}{2} .
$$

Thus the vertex of the parabola (10) has an $i$-coordinate less than $\frac{1}{2}$. Hence, if both solutions of (10) are positive, they are both smaller than one. We note, further, that if a solution $i^{*}$ of (10) falls within the range $(0,1)$, then the corresponding values $s^{*}, v^{*}, r^{*}, \int_{0}^{\infty} u(\theta) d \theta$ given by (9) do so as well, since they are nonnegative (by inspection of (9)) and satisfy (2).

We can observe that these endemic equilibria move further from each other as $\mathcal{R}(\psi)$ increases (and $\psi$ decreases) by differentiating (10) implicitly with respect to $\psi$ or $\mathcal{R}(\psi)$. In either case the numerator of the partial of $i$ has a constant sign (negative with respect to $\psi$ and positive with respect to $\mathcal{R}(\psi)$ ), since both $B$ and $C$ are increasing with respect to $\psi$ as long as $\mathcal{R}(\psi)<1$; the denominator, however, contains $2 A i+B$, which is $\pm \sqrt{B^{2}-4 A C}$ for the two solutions. Therefore, the partials' signs differ.

We here give a specific example, to illustrate the possibility that the hypotheses of Proposition 1 can be fulfilled. We consider an application to feline calicivirus using the following parameter values adapted from Reade et al. [19]: $b=1 / 1095$ days $^{-1}, \psi=0.001$ days $^{-1}, \sigma=0.5, k=1 / 30$ days $^{-1}$, $\delta(\theta)=.000194$ days $^{-1}, \alpha(\theta)=1 / 45$ days $^{-1}, c=1000$ days $^{-1}$, and allowing $\gamma$ to vary to create the

\footnotetext{
${ }^{1}$ The constant coefficient $C$ in equation $(10)$ is, of course, not to be confused with the state variable $C(t)$ in $(1)$.
} 


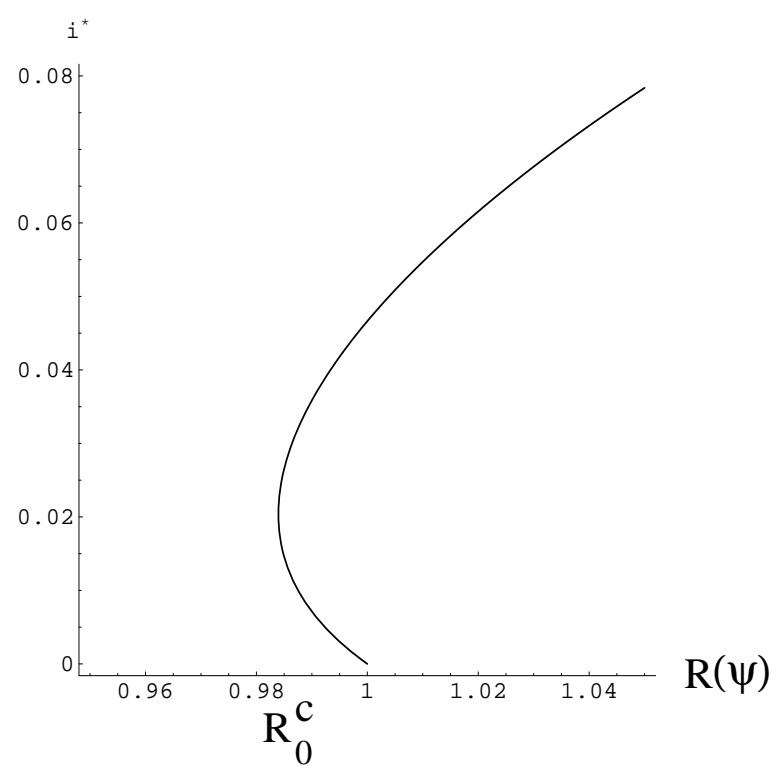

Figure 1: Bifurcation diagram for model (3) showing backward bifurcation in an application to FCV, with parameters as given in text

graph shown in Figure 1. For values of $\gamma$ that make $\mathcal{R}(\psi) \approx \mathcal{R}_{0}^{c}$, we get an acute endemic level $i^{*}$ of about $2 \%$; for $\gamma$ such that $\mathcal{R}(\psi) \approx 1$, the [upper] endemic equilibrium is at about $5 \%$. We note, however, that this range of values for $\gamma$ is about two orders of magnitude higher than the value of 0.000339 adapted from [19], so this particular illustration would require a significant rise in infection rate in order for $\mathcal{R}(\psi)$ to enter the range where multiple endemic equilibria exist. This example also has a high value for $c$, which may lead one to speculate that a similar SIS model can also exhibit multiple endemic states; we shall consider this and a number of other special cases in the next section.

It is useful to put the criteria derived above in terms of the model parameters in order to understand them better. From the point of view of a vaccination campaign, we may be most interested in $\psi$. We can rewrite the three conditions of Proposition 1 in terms of $\psi$, to see that there is a range of values of $\psi$ which can satisfy the conditions. First, however, we must assume that $\sigma \gamma<b+k$, or else it will not be possible to reduce $\mathcal{R}(\psi)$ below one no matter how high $\psi$ is (the minimum value of $\mathcal{R}(\psi)$ is $\mathcal{R}(\infty)=\frac{\sigma \gamma}{b+k}$, the reproductive number when everyone is vaccinated).

$$
\begin{gathered}
\mathcal{R}(\psi)<1 \text { corresponds to } \psi>b \frac{\gamma+d k-(b+k)}{b+k-\sigma \gamma} ; \\
B<0 \text { corresponds to } \psi<\frac{\gamma+d k-(b+k)}{\left(1+\frac{k}{b+c}(1+c \tau)\right)}-b \frac{(\gamma+d k)}{\sigma \gamma} ; \\
B^{2}-4 A C>0 \text { corresponds to } \psi^{2}+2\left[K-2 \frac{b+k}{\sigma \gamma} \frac{\gamma+d k-\sigma \gamma}{\left(1+\frac{k}{b+c}(1+c \tau)\right)}\right] \psi+K^{2}>0,
\end{gathered}
$$

where

$$
K=\frac{\gamma+d k-(b+k)}{\left(1+\frac{k}{b+c}(1+c \tau)\right)}+b \frac{\gamma+d k}{\sigma \gamma}
$$


Note that if $\mathcal{R}_{0}<1$ then $\gamma+d k-(b+k)<0$, making the first condition trivial to satisfy that is, vaccination is not necessary to reduce $\mathcal{R}(\psi)$ below one - but the second impossible. In fact, in the case $\mathcal{R}_{0}<1$, not only is a backward bifurcation not possible, but the DFE is globally stable.

Proposition 2. If $\mathcal{R}_{0}<1$ then the disease-free equilibrium of (3) is globally asymptotically stable.

Proposition 2 rules out backward bifurcation in the case of $\mathcal{R}_{0}<1$. Epidemiologically, this means that if the disease's reproductive number is below one in the absence of vaccination, then vaccination can only contribute to further reduction of the reproductive number; there is no potential danger for hysteresis in the prevalence numbers.

The proof of Proposition 2 is in Appendix 1.

\section{$3 \quad$ Special cases}

We will now consider some special cases of the model(s) developed in the previous section. Some of these are of interest in modeling diseases with different life cycles, while others will provide insights into the original model(s). We will concentrate on the effects on the criteria developed above for multiple endemic equilibria, as without them the vaccination question reduces to making $\psi$ high enough that $\mathcal{R}(\psi)<1$.

\subsection{No variability by infection age}

Let us first consider the case where $\delta$ and $\alpha$ are constants. This will reduce the system to a set of ODEs, and eliminate the integral expressions in the criteria developed. (The equations for $u(\theta, t)$ are replaced by an equation for $U(t) \equiv \int_{0}^{\infty} u(\theta, t) d \theta$.) We find that $\pi(\theta)=e^{-\alpha \theta}$, that $\tau=\frac{1}{b+\alpha}$, and that $d=\frac{\delta}{b+\alpha}$, paralleling the expression $\frac{\gamma}{b+k}$ found in $\mathcal{R}(\psi)$.

This case also allows a straightforward bifurcation analysis via the technique of center manifolds and normal forms (see, for instance, $[16,21]$ ). A lengthy computation following the standard approach yields the following necessary and sufficient criterion for the existence of a backward bifurcation at $\mathcal{R}(\psi)=1$ (see Appendix 2 for details):

$$
\sigma \gamma \frac{\psi}{b+\psi}(\gamma(1-\sigma)+d k)>(b(\gamma+d k)+\sigma \gamma \psi)\left(1+\frac{k}{b+c}(1+c \tau)\right) .
$$

This technique also verifies the local stability of the endemic equilibrium for small $i^{*}$ in case of a forward bifurcation, and its instability for small $i^{*}$ in case of a backward bifurcation.

Note that although the conditions for multiple endemic equilibria given in Proposition 1 imply this criterion, it is not equivalent to them, since the existence of the additional equilibria requires not only that the bifurcation be backward, but that $\mathcal{R}_{0}^{c}<\mathcal{R}(\psi)<1$ (see, e.g., Figure 1). We also see that here, as before, sufficiently extreme (high or close to zero) values of $\psi$ preclude the criterion from holding, so it can only hold for a range of vaccination rates.

This verifies that the multiple endemic equilibria exhibited by model (3) are indeed caused by a backward bifurcation.

\subsection{SIS models, and models with no chronic stage}

Some diseases, such as hepatitis B, do not confer immunity after recovery. We can consider an SIS version of the model (no temporary immunity) by taking $c \rightarrow \infty$. This yields the model 


$$
\begin{aligned}
s^{\prime} & =b(1-s)-\gamma s i-s \int_{0}^{\infty} \delta(\theta) u(\theta, t) d \theta-\psi s+\int_{0}^{\infty} \alpha(\theta) u(\theta, t) d \theta \\
v^{\prime} & =\psi s-\sigma \gamma v i-b v \\
i^{\prime} & =\gamma i(s+\sigma v)+s \int_{0}^{\infty} \delta(\theta) u(\theta, t) d \theta-(b+k) i \\
u_{\theta}+u_{t} & =-(b+\alpha(\theta)) u(\theta, t) ; \quad u(0, t)=k i
\end{aligned}
$$

The only change in the criteria for multiple endemic equilibria is that the expression $\left(1+\frac{k}{b+c}(1+c \tau)\right)$ reduces to $1+k \tau$. As can be seen from (11), $\tau<1 / b$, so that the timescale $\tau$ of the disease is faster than the demographic timescale $1 / b$, and $b \tau<1$. This makes the expression $\left(1+\frac{k}{b+c}(1+c \tau)\right)$ monotone decreasing in $c$, so that as $c \rightarrow \infty$ the value of $B$ in (10) decreases, making it more likely that $B<0$; further, if $B<0$, then $B^{2}-4 A C$ is an increasing function of $c$, so that in general the criteria are easier to fulfill for the model above. Also, the right hand side of (12) decreases, making that criterion easier to fulfill as well. Therefore elimination of the $R$ class facilitates the existence of backward bifurcations in model (3).

If we further eliminate the chronic stage by taking $\alpha(\theta) \rightarrow \infty$ (which causes $d \rightarrow 0$ ), we obtain a simpler SIS model which resembles those of [7] and [17]. Consistent with the results of those papers, backward bifurcation is possible here as well. A general comparison of parameter ranges is difficult since the criteria of Proposition 1 may be easier or harder to satisfy, depending on values of the parameters.

We can also develop SIRS models without chronic stages, either by taking $c \rightarrow \infty$ and $\delta=0$ (effectively converting the chronic stage into a recovered stage with variable recovery rate) or by simply letting $\alpha(\theta) \rightarrow \infty$ without changing $c$. In these cases also, the criteria for multiple endemic equilibria only change quantitively rather than qualitatively, and can still be met.

Comparison of SIRS and SIS models with and without chronic stages shows that the existence of a chronic stage neither makes possible nor makes impossible the existence of multiple endemic equilibria via a backward bifurcation, although it does, of course, increase $\mathcal{R}(\psi)$. However, recall that with the chronic stage present, a vaccine which provides protection against infection by chronicstage infectives, but no protection against infection by acute infectives $(\sigma=1)$, may still allow backward bifurcations. With the chronic stage absent, this distinction is lost, and a vaccine with $\sigma=1$ does not effectively separate the susceptible pool into two groups; therefore no backward bifurcation is possible.

\subsection{An SIR model}

If we take $c=0$, on the other hand, we have an SIR model in which the only renewal of the susceptible pool is through newborns. This corresponds to diseases like hepatitis A which confer permanent immunity following recovery, but not infections such as herpes, in which both vaccination and recovery from infection result in permanent low-level virus presence in the body, and in which case one would model recovery from infection directly into the vaccinated class.

In this case, we find that the condition $\mathcal{R}(\psi)<1$ precludes $B<0$ (though $\mathcal{R}(\psi)$ is independent of $c, B$ is not), through the following sequence of implications:

$$
R(\psi)=\frac{b(\gamma+d k)+\sigma \gamma \psi}{(b+\psi)(b+k)} \quad<1
$$




$$
\begin{aligned}
\frac{1}{b+k} & <\frac{b+\psi}{b(\gamma+d k)+\sigma \gamma \psi} \\
\frac{b(\gamma+d k)}{b+k} & <\frac{b(\gamma+d k)}{b(\gamma+d k)+\sigma \gamma \psi}(b+\psi)<(b+\psi) \\
\frac{b}{b+k}(\gamma+d k) & <b+\psi+\frac{b(\gamma+d k)}{\sigma \gamma}=b+\frac{b(\gamma+d k)+\sigma \gamma \psi}{\sigma \gamma} \\
\sigma \gamma(\gamma+d k) & <\sigma \gamma(b+k)+\left(\frac{b+k}{b}\right)(b(\gamma+d k)+\sigma \gamma \psi) \\
0 & <(b(\gamma+d k)+\sigma \gamma \psi)\left(\frac{b+k}{b}\right)+\sigma \gamma(b+k-(\gamma+d k))=B
\end{aligned}
$$

Therefore there are no backward bifurcations possible. From this we see that the demographic renewal process of births and deaths is insufficient to permit multiple endemic equilibria - the disease cycle itself must be closed (infectives eventually become susceptible again). This result contrasts with the well-known analysis of the simplest possible model for an SIR epidemic, in which the inclusion of birth-death demographics makes a significant change in the long-term behavior of the disease (i.e., oscillations of very long period, compared to a single outbreak which completely dies out). For backward bifurcations, at least, it does not make the crucial difference.

Note that if we cut the disease cycle off even earlier, setting $\alpha(\theta)$ or $k$ to zero to create an SI model (with or without chronic stage, respectively), we again find that no backward bifurcations are possible, for precisely the same reason as with the SIR model. Neither of these models is appropriate for HIV infection as there is no removal to full-blown AIDS.

\subsection{Slow external demographics}

To consider again the role of the birth-death process in the existence of a backward bifurcation, we can let $b \rightarrow 0$. The interpretation here is that the disease time-scale is considerably faster than the time-scale of demographics external to the disease. Re-examining the criteria for multiple endemic equilibria given in Proposition 1, we see that backward bifurcations are still possible given sufficiently small $\psi$. One way to account for this is to note that for $b<<\psi$, i.e., the demographic time-scale is even slower than that of the vaccination campaign, we find that almost everyone is vaccinated, so the susceptibles are not effectively divided into distinct groups $(s<<v)$. From a disease control perspective this means that vaccinated individuals remain in the population, rather than being replaced through mortality with unvaccinated newborns. This does not, however, mean that fast diseases are easier to control: the reverse is usually true. For purposes of disease control one needs to distinguish between the rates of disease transmission and vaccination, as well as with regard to natural mortality rates. In this case it is the disparity between the time-scales of external demographics and the vaccination campaign that preserves the possibility of backward bifurcation, rather than that between the time-scales of external demographics and infection.

\section{Effects of variability}

We have seen above that the effects of the variability of $\alpha(\theta)$ and $\delta(\theta)$ on $\mathcal{R}(\psi)$ and the possibility of backward bifurcation are restricted to their appearances in the expressions for $d(7)$ and $\tau$ (11). In order to separate the effect of variability from the effect of scale, we can consider the question of whether two distinct functions with the same "average" value yield the same values for $d$ and $\tau$. We will focus first on $\alpha$. 
A reasonable way to define the effect of the scale (magnitude) of $\alpha(\theta)$ is to consider the average time to recovery (ignoring other factors such as mortality), given by

$$
T(\alpha) \equiv \int_{0}^{\infty} \pi(\theta) d \theta
$$

since $\pi(\theta)$ represents the probability of remaining infected a time $\theta$ after having entered the (chronic) infective compartment. If we now consider $b$ fixed and $\tau$ as a function $\tau(\alpha)$ of $\alpha$, we can ask whether it is possible to have two different functions $\alpha_{1}(\theta)$ and $\alpha_{2}(\theta)$ which satisfy the criteria set forth in Section 2, and such that $T\left(\alpha_{1}\right)=T\left(\alpha_{2}\right)$ and $\tau\left(\alpha_{1}\right)=\tau\left(\alpha_{2}\right)$.

It $i s$ possible to have such functions, and we here provide an example. Let

$$
f(x) \equiv\left[\frac{1}{6}(b+1) x^{2}-\frac{1}{2}(b+2) x+1\right] x e^{-x} .
$$

$f$ has the properties that $f(0)=0, \int_{0}^{\infty} f(x) d x=0$, and $\int_{0}^{\infty} f(x) e^{-b x} d x=0$. Therefore, if we have two functions $\alpha_{1}$ and $\alpha_{2}$ such that the corresponding $\pi_{1}$ and $\pi_{2}$ differ by a multiple of $f, M f$, the linearity of the functionals $T$ and $\tau$ gives us

$$
T\left(\alpha_{1}\right)-T\left(\alpha_{2}\right)=T\left(\alpha_{1}-\alpha_{2}\right)=T(M f)=M T(f)=0, \text { so that } T\left(\alpha_{1}\right)=T\left(\alpha_{2}\right),
$$

and likewise for $\tau$. Furthermore, since $f(0)=0$, we have $\pi_{1}(0)=\pi_{2}(0)[=1]$, as we must. (Here $f$ may represent a difference in recovery rates which is significant for individuals newly arrived into the chronic class, but diminishes with time.)

Of course, it is also necessary that $\pi_{1}$ and $\pi_{2}$ decrease monotonically to zero as $\theta$ increases, fast enough to be $L_{1}[0, \infty)$. A simple example of a pair of such functions is as follows. Take $\alpha_{1}(\theta)=\alpha_{1}$ constant, so that $\pi_{1}(\theta)=e^{-\alpha_{1} \theta}$. Then define $M=\alpha_{1} e^{-\alpha_{1} x_{0}} / \max _{\theta} f^{\prime}(\theta)>0$, where $x_{0}$ is the greater of $f$ 's two local maxima (for $x>x_{0}, f(x)$ is decreasing), and let $\pi_{2}(\theta) \equiv \pi_{1}(\theta)+M f(\theta)$. $\pi_{2}$ decreases monotonically from 1 at $\theta=0$ to 0 ; to see the monotonicity we observe that $\pi_{2}^{\prime}(\theta)=\pi_{1}^{\prime}(\theta)+M f^{\prime}(\theta)$. For $\theta \geq x_{0}, f^{\prime}(\theta) \leq 0$, so $\pi_{2}^{\prime}(\theta)$ is clearly negative. For $0 \leq \theta<x_{0}$,

$$
\pi_{2}^{\prime}(\theta)=-\alpha_{1} e^{-\alpha_{1} \theta}+\alpha_{1} e^{-\alpha_{1} x_{0}} \frac{f^{\prime}(\theta)}{\max _{\theta} f^{\prime}(\theta)}<\alpha_{1}\left(-e^{-\alpha_{1} \theta}+e^{-\alpha_{1} x_{0}}\right)<0 .
$$

Therefore there are cases in which the form of $\alpha$ affects neither $\mathcal{R}(\psi)$ nor the possibility of backward bifurcation. In particular, the above example shows that a constant $\alpha$ can have the same effect as a variable one.

There are also many T-comparable $\alpha$ 's which do produce different values of $d$ and $\tau$. We may consider briefly the two most commonly-used examples, also often thought to be opposite extremes with regard to stability of equilibria (cf. [15]): namely, an exponential decay (corresponding to constant $\alpha$ ) and a fixed-delay step function ${ }^{2}$. If we fix $T$ at $T^{*}$, then the exponential decay is $\pi_{e}(\theta)=e^{-\theta / T^{*}}$ (we note that if $\alpha$ is constant, $T^{*}=1 / \alpha$ ), and the step function $\pi_{s}(\theta)$ is 1 for $\theta \in\left[0, T^{*}\right]$ and 0 elsewhere. The resulting values of $\tau$ are

$$
\tau_{e}=\frac{T^{*}}{b T^{*}+1}<\tau_{s}=\frac{1-e^{-b T^{*}}}{b},
$$

which means that time spent in the chronic stage is less for an exponentially decaying probability $\pi_{e}$ than for a step-function probability $\pi_{s}$ with equivalent $T$ value. Furthermore, if we take $\delta$ constant,

\footnotetext{
${ }^{2}$ Although technically a step function $\pi$ cannot arise from a finite $\alpha$, an arbitrarily small perturbation can resolve the problem.
} 
this means that the step-function probability gives a greater value for $d$, and thus for $\mathcal{R}(\psi)$, than if $\alpha$ were constant.

In order to address the effects of the form of $\delta(\theta)$, we also need to develop the notion of an "average" value for $\delta$. However, here the way to do so is not as clear. We might, for instance, define $\bar{\delta} \equiv \lim _{t \rightarrow \infty} \int_{0}^{t} \delta(\theta) d \theta / t$, which reduces to $\lim _{\theta \rightarrow \infty} \delta(\theta)$ if it exists, or we might take instead $\hat{\delta} \equiv \int_{0}^{\infty} \delta(\theta) \pi(\theta) d \theta$. We can easily find two $\bar{\delta}$-comparable functions which yield different values for $d$ (given the same $\alpha$ ); if we take $\alpha$ constant, then we can also find two $\hat{\delta}$-comparable functions which yield different values for $d$, by the method given above involving $f(x)$.

\section{Discussion}

Our goal in this investigation has been to explore factors affecting the possibility of multiple endemic equilibria, with a focus on disease control via a vaccination program in which the vaccine provides complete protection against low-level infection and partial protection against infection by acute infectives. In order to be of any use, of course, the vaccine must have sufficient potential to reduce the disease's ability to spread $(\mathcal{R}(\infty)=\sigma \gamma /(b+k)<1)$. Given such potential, a sufficiently high vaccination rate will reduce $\mathcal{R}(\psi)$ below one. The additional concern is that such reduction may not be sufficient to eradicate the disease, or prevent an outbreak from spreading, if conditions allow a backward bifurcation. In general, endemic equilibria can be avoided by further increasing $\psi$ so that the condition $B<0$ cannot be met, as given at the end of Section 2 .

Since vaccination campaigns cost money, we may wish to consider the problem of minimizing $\mathcal{R}(\psi)$ relative to a cost constraint. However, since $\mathcal{R}(\psi)$ is monotone decreasing in $\psi$, we should just increase $\psi$ until we either reach our maximum allowable cost or cross the threshold value for $\psi$, which depends on the characteristics of the disease and the efficiency $\sigma$ of the vaccine. The latter threshold value for $\psi$ may be given by the condition $\mathcal{R}(\psi)=1$, or by $B=0$ if conditions cause a backward bifurcation. If, on the other hand, we wish to minimize the cost associated with a vaccination campaign, again we must first estimate other system parameters, and then increase $\psi$ until it reaches whichever of these two thresholds applies.

The special cases we considered in Section 3 showed that the possibility of a backward bifurcation exists here only for closed disease cycles (SIRS and SIS). SIR and SI models do not allow multiple endemic states because they preclude a rapid infection-recovery cycle that can enable a disease to take hold of part of a population, while the rest (in this case, the vaccinated) remain relatively stable and unaffected (cf. [7, 17]). This is true even when natural demographics are a significant source of renewal for the susceptible pool. Indeed, more generally we can see that for sufficiently small values of $c, k$ or $\alpha$, no backward bifurcations are possible. That is, if progression from acute to chronic stage, or recovery, or loss of immunity, is slow enough, it is impossible for the disease cycle to establish itself in any part of the population as long as $\mathcal{R}(\psi)<1$.

Exceptional features of the models considered above include a chronic infective class with infectivity and recovery rates which vary with age of [chronic-stage] infection. We have seen that the existence of a chronic stage does not qualitatively affect the possibility of backward bifurcations, except in the case of a vaccine which protects only against the low-level infectivity of chronic-stage individuals, where multiple endemic states may be possible. Of course, the secondary infection mechanism does increase $\mathcal{R}(\psi)$. Hadeler and Castillo-Chavez [7] examined a model for an infection without a chronic stage which exhibited a backward bifurcation under certain conditions, but in which a proportion of recovering infectives enter the vaccinated class. Castillo-Chavez and Huang [2] considered a model structured by age of individual in which the variability of contact rates by age, together with prevalence-dependent recruitment rates, permitted a backward bifurcation 
to occur in some cases. In our model(s) the infectivity and recovery rates which vary by age of infection have a similar interaction with the vaccination program.

It has been seen (for example, in [15]) that the form of the function describing the rate at which individuals recover from a disease can affect the disease's long-term behavior; for instance, fixed-duration infections may be more likely to generate sustained oscillations than the customary exponential recovery rate. Brauer found that variable infectivity in some SIR and SIS models could affect the stability of endemic equilibria for $\mathcal{R}(\psi)>1$. In this model we have assumed an exponential rate of progress from acute to chronic stage, but allowed the per capita infectivity and recovery rates of the chronic stage to vary. We have seen that for models (1) and (3) the forms of $\alpha(\theta)$ and $\delta(\theta)$ are only relevant to backward bifurcation as they appear in the expressions for $d(7)$ and $\tau$ (11). There are comparable but distinct forms that have exactly the same effect on system behavior, including constant vs. variable $\alpha$, but there is a difference between the forms of $\pi(\theta)$ most commonly used - in particular, chronic infections of fixed duration may cause a higher value for $\mathcal{R}(\psi)$ than infections with a constant per capita recovery rate (and the same average duration of infection).

\section{Appendix 1. Proof of Proposition 2}

Proposition 2. If $\mathcal{R}_{0}<1$ then the disease-free equilibrium of (3) is globally asymptotically stable.

Proof. One can integrate the fourth equation of (3) along the characteristic lines to obtain:

$$
u(\theta, t)= \begin{cases}u_{0}(\theta-t) e^{-b t} \pi(\theta-t, \theta) & \theta \geq t \\ k i(t-\theta) e^{-b \theta} \pi(\theta) & \theta<t\end{cases}
$$

where $\pi(\theta-t, \theta)=e^{-\int_{\theta-t}^{\theta} \alpha(\eta) d \eta}$ and $u(\theta, 0)=u_{0}(\theta)$. Since $\alpha(\theta)$ and $\delta(\theta)$ are bounded functions, let

$$
\bar{\delta}=\sup _{\theta \in[0, \infty)} \delta(\theta) \quad \bar{\alpha}=\sup _{\theta \in[0, \infty)} \alpha(\theta) .
$$

We can estimate the behavior at infinity of the force of infection generated by the chronic individuals:

$$
\begin{aligned}
\int_{0}^{\infty} \delta(\theta) u(\theta, t) d \theta & =\int_{0}^{t} \delta(\theta) k i(t-\theta) e^{-b \theta} \pi(\theta) d \theta+\int_{t}^{\infty} \delta(\theta) u_{0}(\theta-t) e^{-b t} \pi(\theta-t, \theta) d \theta \\
& \leq \int_{0}^{t} \delta(\theta) k i(t-\theta) e^{-b \theta} \pi(\theta) d \theta+\bar{\delta}\left\|u_{0}\right\|_{L^{1}} e^{-b t}
\end{aligned}
$$

We can now take limsup as $t \rightarrow \infty$ of both sides of this inequality. The integral on the second line is of convolution type. We can move the limsup inside the integral since $i(t)$ is nonnegative and bounded and the convolution kernel is nonnegative and assumed integrable. Note that $\lim \sup _{t} \bar{\delta}\left\|u_{0}\right\|_{L^{1}} e^{-b t}=0$. Therefore, from (13) we obtain

$$
\limsup _{t} \int_{0}^{\infty} \delta(\theta) u(\theta, t) d \theta \leq \int_{0}^{\infty} \delta(\theta) k e^{-b \theta} \pi(\theta) d \theta \cdot \limsup _{t} i(t)=k d \limsup i(t) .
$$

Furthermore, if we integrate the third equation in (3), we observe that $s(t)+\sigma v(t) \leq 1$ for all $t$ and take limsup. Applying the inequality above, we conclude

$$
\underset{t}{\limsup } i(t) \leq \frac{1}{b+k} \limsup _{t}\left[\gamma(s(t)+\sigma v(t)) i(t)+s(t) \int_{0}^{\infty} \delta(\theta) u(\theta, t) d \theta\right]
$$




$$
\begin{aligned}
& \leq \frac{1}{b+k}[\gamma+k d] \limsup i(t) \\
& =\mathcal{R}_{0} \limsup _{t} i(t) .
\end{aligned}
$$

Since $\mathcal{R}_{0}<1$, this inequality is only true if $\limsup _{t} i(t)=0$. Consequently, $\lim _{t} i(t)=0$. Computation similar to (14) [but without $\delta$ ] shows that $\int_{0}^{\infty} u(\theta, t) d \theta \mapsto 0$, which implies that $u(\theta, t) \mapsto 0$ for almost all $\theta$. Assuming $\alpha(\theta)$ is bounded, from the equation for the recovered class in (3) we see that $\lim _{t} r(t)=0$. Furthermore, from the first equation in (3) we have $s^{\prime} \leq b-(b+\psi) s$. Integrating this inequality and taking limsup we get

$$
\limsup _{t} s \leq \frac{b}{b+\psi} .
$$

Next, from the second equation in (3), disregarding the second term in the right hand side, we obtain the inequality $v^{\prime} \leq \psi s-b v$. Integrating, taking lim sup and using (15) we see that

$$
\limsup _{t} v=\frac{\psi}{b} \limsup _{t} s \leq \frac{\psi}{b+\psi} .
$$

Taking a limsup in equation (2) we see that

$$
1 \leq \limsup _{t} s+\limsup _{t} v \leq \frac{b}{b+\psi}+\frac{\psi}{b+\psi}=1 .
$$

Therefore, all inequalities are, in fact, equalities. Thus,

$$
\limsup _{t} s=\frac{b}{b+\psi}, \quad \quad \limsup v=\frac{\psi}{b+\psi} .
$$

In addition, taking the liminf of equation (2) we obtain the same values for the $\liminf _{t} s$ and $\liminf \operatorname{in}_{t}$. Consequently,

$$
\lim _{t} s=\frac{b}{b+\psi}, \quad \quad \lim _{t} v=\frac{\psi}{b+\psi} .
$$

\section{Appendix 2. Bifurcation analysis}

We here provide a few details of the analysis of the bifurcation at $\mathcal{R}(\psi)=1$ mentioned in Section 3.1. To reduce complexity of terms, we give below the results for $c \rightarrow \infty$, but neither $\mathcal{R}(\psi)$ nor the corresponding bifurcation parameter changes, and the result extends naturally to the more general case. In this case, following the standard technique (e.g., in [16]), we find that the dynamics on the center manifold are

$$
x^{\prime}=\frac{(b+\alpha)^{2}(b+\psi)}{(b+\alpha)^{2}(b+\psi)+\delta k b}\left[(b+k) p x-f x^{2}\right]+\mathcal{O}(3),
$$

where

$$
\begin{gathered}
x \equiv\left(i-\left(b+\alpha+\lambda_{2}\right) U / k\right) /\left(\lambda_{1}-\lambda_{2}\right), \\
p \equiv \mathcal{R}(\psi)-1 \text { is the bifurcation parameter, }
\end{gathered}
$$




$$
\begin{gathered}
f \equiv[\gamma+d k](b+\alpha+k)-[(1-\sigma) \gamma+d k]\left[(b+\alpha+k)+\sigma \gamma \frac{b+\alpha}{b+\psi}\right] \frac{\psi}{b+\psi}, \\
\lambda_{1}, \lambda_{2} \equiv \frac{1}{2}\left\{-F \pm \sqrt{F^{2}+4(b+\alpha)(b+k) p}\right\}, \quad F \equiv\left[\frac{(b+\alpha)^{2}(b+\psi)+\delta k b}{(b+\alpha)(b+\psi)}-(b+k) p\right],
\end{gathered}
$$

and $\mathcal{O}(3)$ refers to third-order and higher terms in $x$ and $p$. Note that

$$
\lambda_{1}-\lambda_{2}=\frac{(b+\alpha)^{2}(b+\psi)+\delta k b}{(b+\alpha)(b+\psi)}+\mathcal{O}(p)>0 ;
$$

thus (from the definition of $x$ ) the direction of increase in $i$ is the same as that of increase in $x$.

From equation (16) we see that the bifurcation at $\mathcal{R}(\psi)=1(p=0)$ is transcritical as long as $f \neq 0$, and is forward in $x$ and $i$ if $f>0$, and backward if $f<0$. We can rewrite the criterion $f<0$ as

$$
\sigma \gamma \frac{\psi}{b+\psi}(\gamma(1-\sigma)+d k)>(b(\gamma+d k)+\sigma \gamma \psi)\left[1+\frac{k}{b+\alpha}\right],
$$

which is implied by the conditions for multiple endemic equilibria given in Proposition 1 . Note that it is not equivalent to them, as a backward bifurcation may exist without multiple endemic equilibria, if $\mathcal{R}(\psi)$ is not in the necessary range $\mathcal{R}_{0}^{c}<\mathcal{R}(\psi)<1$ (see Figure 1 ).

The final term $1+k /(b+\alpha)$ in the expression above becomes $1+\frac{k}{b+\alpha}\left(1+\frac{\alpha}{b+c}\right)$ for finite $c$, and corresponds to the expression $\left(1+\frac{k}{b+c}(1+c \tau)\right)$ seen in the coefficients of $(10)$.

\section{Acknowledgments}

CMKZ wishes to thank Hristo Kojouharov for several very helpful conversations, as well as the loan of a copy of [13], and Antonio Behn for suggesting part of the example in Section 4. MM thanks Tanya Kostova for comments on an early draft of this work. The authors thank the referees for their helpful suggestions.

\section{References}

[1] F. Brauer, Variable infectivity in communicable disease models, in Proceedings of the First World Congress of Nonlinear Analysts, Tampa, Florida, August 19-26, 1992 (V. Lakshmikantham, ed.), Walter de Gruyter, New York, 1996, pp. 3201-3210.

[2] C. Castillo-Chavez \& W. Huang, Age-structured core group model and its impact on STD dynamics, in Mathematical Approaches for Emerging and Reemerging Infectious Diseases: Models, Methods and Theory, IMA Volume 126 (C. Castillo-Chavez, S. Blower, D. Kirschner, P. van den Driessche \& A.-A. Yakubu, eds.), Springer-Verlag, Berlin, 2001, pp. 261-273.

[3] Z. Feng, C. Castillo-Chavez, \& A. Capurro, A model for tuberculosis with exogeneous reinfection, Theor. Pop. Biol. 57: 235-247, 2000.

[4] D. Greenhalgh, O. Diekmann, \& M. C. M. de Jong, Subcritical endemic steady states in mathematical models for animal infections with incomplete immunity, Math. Biosci. 165(1): $1-25,2000$. 
[5] K. Hadeler, Pair formation with maturation period, J. Math. Biol. 32: 1-15, 1993.

[6] K. Hadeler, Periodic solutions of homogeneous equations, Journal of Differential Equations 95: 183-202, 1992.

[7] K. Hadeler \& C. Castillo-Chavez, A core group model for disease transmission, Math. Biosci. 128: 41-55, 1995.

[8] K. Hadeler \& J. Müller, Vaccination in age structured population I: The reproduction number, in Epidemic models: Their structure and relation to data (V. Isham and G. Medley, eds.), Cambridge University Press, Cambridge, 1996.

[9] K. Hadeler \& J. Müller, Vaccination in age structured population II: Optimal vaccination strategies, in Epidemic models: Their structure and relation to data (V. Isham and G. Medley, eds.), Cambridge University Press, Cambridge, 1996.

[10] K. Hadeler \& P. van den Driessche, Backward bifurcation in epidemic control, Math. Biosci. 146: 15-35, 1997.

[11] K. Hadeler \& R. Waldstätter, A. Wörz-Busekros, Models for pair formation in bisexual populations, J. Math. Biol. 26: 635-649, 1988.

[12] W. Huang, K. Cooke, \& C. Castillo-Chavez, Stability and bifurcation for a multiple-group model for the dynamics of HIV transmission, SIAM J. Appl. Math. 52(3): 835-854, 1992.

[13] M. Iannelli, Mathematical theory of age-structured population dynamics, Applied Mathematics Monographs 7, Giardini Editori, Pisa, 1995.

[14] M. Y. Kim \& F. Milner, A mathematical model of epidemics with screening and variable infectivity, Math. Comput. Modelling 21(7): 29-42, 1995.

[15] C. M. Kribs-Zaleta, Toward quantifying the spectrum of recovery functions, Dynamics of Continuous, Discrete and Impulsive Systems 7(1): 1-17, 2000.

[16] C. M. Kribs-Zaleta, Center manifolds and normal forms in epidemic models, in Mathematical Approaches for Emerging and Reemerging Infectious Diseases: Models, Methods and Theory, IMA Volume 125 (C. Castillo-Chavez et al., eds.), Springer-Verlag, Berlin, 2001, pp. 269-286.

[17] C. M. Kribs-Zaleta \& J. X. Velasco-Hernández, A simple vaccination model with multiple endemic states, Math. Biosci. 164: 183-201, 2000.

[18] H. Thieme \& C. Castillo-Chavez, How may infection-age-dependent infectivity affect the dynamics of HIV/AIDS?, SIAM J. Appl. Math. 53(5): 1447-1479, 1993.

[19] B. Reade, R. Bowers, M. Begon, R. Gaskell, A model of disease and vaccination for infection with acute and chronic phases, J. Theor. Biol. 190: 355-367, 1998.

[20] S. Tu \& E. Ross, Minimum transition values and the dynamics of subcritical bifurcation, SIAM Journal of Applied Mathematics 46(2): 189-198, 1986.

[21] S. Wiggins, Introduction to applied nonlinear dynamical systems and chaos, Springer-Verlag, New York, 1990. 